\title{
Effet De Fumures Minérales Sur Le Rendement Et La Qualité Organoleptique Du Manioc (Manihot Esculenta Crantz) Dans La Zone De Savane Au Centre-Sud De Centrafrique
}

\section{Christiant Simplice Armand Ballot, Doctorant}

Laboratoire des Sciences Biologiques et Agronomiques pour le

Développement, Faculté des Sciences, Université de Bangui, République Centrafrique

Laboratoire Botanique Écologie Végétale, Faculté Des Sciences, Université de Lomé, Lomé Togo

Silla Semballa, Maitre-assistant

Laboratoire des Sciences Biologiques et Agronomiques pour le

Développement, Faculté des Sciences, Université de Bangui, République Centrafrique

Wouyo Atakpama, Assistant

Laboratoire Botanique Écologie Végétale,

Faculté Des Sciences, Université de Lomé, Lomé Togo

Tatiana Maryse Yangakola, Doctorante Arnaud Doubro Bo-Mbi, Doctorant

Laboratoire des Sciences Biologiques et Agronomiques pour le Développement, Faculté des Sciences, Université de Bangui, République Centrafrique

Didier Blavet, Chargé de Recherches

UMR Eco\&Sols IRD, Montpellier France

Innocent Zinga, Assistant

Laboratoire des Sciences Biologiques et Agronomiques pour le

Développement, Faculté des Sciences,

Université de Bangui, République Centrafrique

Kpérkouma Wala, Maitre de Conférences

Kolman Batawila, Professeur Titulaire

Koffi Akpagana, Professeur Titulaire

Laboratoire Botanique Écologie Végétale,

Faculté Des Sciences, Université de Lomé, Lomé Togo 


\section{Abstract}

Aims - The farming intensification in Sub-Saharan Africa induced soil fertility and crop yields depletion such as cassava, a main food and an important source of income of farmers in Central Africa Republic. To transcend the soil fertility depletion and improve cassava yield, LaSBAD has initiate a study focused on the mineral fertilizers of cassava crops.

Methods - Four types of fertilizer were tested: the urea $\left(\mathrm{Co}\left(\mathrm{NH}_{2}\right)_{2}\right)$, the potassium sulfate $\left(\mathrm{K}_{2} \mathrm{SO}_{4}\right)$, the potassium chloride $(\mathrm{KCl})$ and the triple superphosphate (TSP). The methodology consisted in the physical and chemical analysis of soil, the cultivation, the application of mineral fertilizers and the harvest of cassava after 12 months. Additionally, the evaluation of the organoleptic propriety of raw tubers and cassava balls after the use of fertlizers were achieved throughout semi-strucrured interviews. Results The contribution of nitrogen and phosphorus has increased very significantly cassava yield from 18.70 to 40.20 t/ha respectively for the control treatment $\left(T_{0}\right)$ and the best treatment obtained $\left(T_{10}\right)$. A significant interaction was observed between nitrogen $(\mathrm{N})$ and potassium $(\mathrm{K})$ inputs on yields. The increasing doses of potassium as potassium chloride (KCl) had reduced cassava plant growth and yield. According to respondents, the organoleptic proprieties of cassava were remain quite unchangeable by mineral fertilizers. Conclusion - The application of mineral fertilizer improved cassava yield and did not affected the organoleptic quality of the raw tubers and cassava ball. Nevertheless, further studies are needed to prevent soil degradation, namely the potential use of termite nest as natural fertilizers and legumes in association or rotation with other crops.

Keywords: Cassava, mineral fertilizers, productivity, savannah, Central Africa Republic.

\section{Résumé}

Objectifs - L’intensification des terres cultivées en Afrique subsaharienne a entrainé une diminution de la fertilité des sols et du rendement de culture telles que celle du manioc, un aliment de base et une importante source de revnu des paysans de la Republique Centrafricaine. Afin de pallier à la diminution de la fertilité des sols et de rendement des cultures du manioc, le LaSBAD s'est fixé comme objectif d'étude, un apport des fumures minérales sur la culture de manioc. Méthodes - Quatre types d'engrais ont été testés : l'urée $\left(\mathrm{Co}\left(\mathrm{NH}_{2}\right)_{2}\right)$, le sulfate de potassium $\left(\mathrm{K}_{2} \mathrm{SO}_{4}\right)$, le chlorure de potassium (KCl) et le triple superphosphate (TSP). La méthodologie a consisté à faire l'analyse physico-chimique des sols, la mise en culture, l'application des fertilisants minérales et la récolte après 12 mois de culture. Une enquête semi-structurée sur l'appréciation organoleptique 
des tubercules crus et de la boule de manioc après l'emploi des fumures sont réalisées . Résultats - L'apport d'azote et de phosphore a permis d'accroître de manière trés significative le rendement de manioc de 18,70 à 40,20 t.ha ${ }^{-1}$ respectivement pour le traitement témoin $\left(\mathrm{T}_{0}\right)$ et le meilleur traitement obtenu $\left(\mathrm{T}_{10}\right)$. Une interaction très significative a été observée entre les apports d'azote $(\mathrm{N})$ et de potassium $(\mathrm{K})$ sur les rendements. L'apport de doses croissantes de potassium sous forme de Chlorure de potassium (KCl) a influencé négativement la croissance des plants et le rendement du manioc. Conclusion - L'application des engrais minéraux a permis d'améliorer de la productivité et n’a pas affecté la qualité organoleptique des tubercules crus et de la boule de manioc. Néanmoins, en perspective d'un développement plus durable en agriculture, d'autres pratiques restent à étudier pour éviter la dégradation des sols, notamment l'utilisation des terres de termitières comme engrais naturels et des légumineuses en association ou en rotation de culture.

Mots-clés: Manioc, fumures minérales, productivité, savane, Centrafrique

\section{Introduction}

La demande alimentaire croissante en Afrique subsaharienne est la conséquence d'intensification agricole et d'augmentation des terres cultivées qui entraînent une diminution de la fertilité des sols ainsi qu'une baisse croissante du rendement des cultures telles que le manioc (Kaho et al., 2011; Milleville and Serpantié, 1994).

En République Centrafricaine l'économie repose sur le secteur agricole qui emploie près de $70 \%$ de la population active et représente $48 \%$ du PIB en 2008. Le manioc (Manihot esculenta Crantz) aliment de base de des centrafricains, représente une importante source de revenus des producteurs du manioc (Zinga et al., 2012). Les résultats de l'analyse globale de la vulnérabilité et de la sécurité alimentaire en RCA en 2009 ont montré que 30,2 \% de la population n’arrivaient pas à satisfaire ses besoins alimentaires de façon adéquate. La production nationale du manioc est en baisse et est estimée à 646000 tonnes de tubercule/an dont 10,8 t ha ${ }^{-1}$ tandis que la demande nationale en manioc ne cesse de croître (Backiny-Yetna and Wodon, 2010; Kafara, 2003; Zinga et al., 2013; Zoundi, 2012).

Afin de pallier au problème d'insécurité alimentaire, le Laboratoire des Sciences Biologiques et Agronomiques pour le Développement (LaSBAD), de l'Université de Bangui (Centrafrique) a initié une étude sur la fertilisation organo-minérale du sol. Cette étude a pour finalité d'accroître le rendement de la culture du manioc et de subvenir aux besoins de la population consommatrice, mais aussi d'améliorer le revenu des paysans producteurs du manioc. En milieu rural, l'utilisation des engrais minéraux en cultures vivrières reste insignifiante compte tenu du faible pouvoir d'achat 
des paysans et de la disponibilité des engrais, l'une des voies prometteuses pour l'amélioration de la productivité du manioc. La technique consiste à apporter aux sols les éléments nutritifs dont les plantes ont besoin à des doses convenables pour accroître les rendements et préserver la capacité de production du sol (Akanza et al., 2002; Howeler, 2011; Palm et al., 2001).

La présente étude, conduite pendant la campagne agricole de 20122013 a pour but d’appliquer des fumures minérales sur la culture du manioc en vue d'augmenter sa productivité et de couvrir les besoins de consommation de la population et améliorer le revenu des paysans producteurs du manioc. Ce qui offrirait la garantie de satisfaction de la demande de la population centrafricaine. Les objectifs spécifiques consistent à : (i) réaliser une analyse physico-chimique des éléments des sols étudiés ; (ii) déterminer la dose optimale et le traitement adéquat recommandé qui répond à la préférence des agriculteurs pour la culture de manioc et (iii) évaluer à travers une enquête ethnosociologique, la qualité organoleptique des tubercules et de la boule de manioc après l'emploi des fumures.

\section{Matériel et méthodes}

\section{Milieu d'étude}

L’étude s'est déroulée sur un site expérimental du LaSBAD, dans le village Ndara 1, situé à $80 \mathrm{Km}$ au nord de Bangui, à une latitude Nord de 0459'622 et une longitude Sud de 1840'442 (Figure 1). C'est un site de champs-écoles réservé aux essais agronomiques pour le renforcement des capacités des producteurs de manioc. Cette zone d'étude est située dans la savane. Le climat est de type Soudano-oubanguien caractérisé par l'alternance de deux saisons : une saison pluvieuse qui s'étend d'avril à septembre et une saison sèche de novembre à janvier, intercalées par une période d’intersaison de février à mars. La pluviométrie annuelle varie de $1200 \mathrm{~mm}$ à $1600 \mathrm{~mm}$ avec une période de croissance des végétaux variant de 240 jours à 180 jours (Bois and Baleux, 2003) . La température moyenne mensuelle varie entre 23 et $27^{\circ} \mathrm{C}$. La végétation est de type savanicole à dominance de : Chromoleana odorata (L.) R.M.King, Panicum maximum Jacq., Pueraria phaseoloides (Roxb.) Benth., Mimosa pudica L., Daniella oliveri (Rolfe) Hutch et Dalziel L., Andropogon gayanus Kunth et Piliostigma thonningii Schumack.. Les sols sont de type ferrallitique moyennement désaturés, moins profonds avec une bonne porosité et une activité biologique intense. D’une façon générale, ils présentent une structure massive et une texture argilo-limono-sableuse. L’activité principale des habitants de la zone est l'agriculture dont les cultures dominantes sont le manioc (M. esculenta), le riz (Oryza sativa L.) et la banane (Musa sapientum L). À côté de cette activité agricole, se pratiquent la pêche et la chasse. 


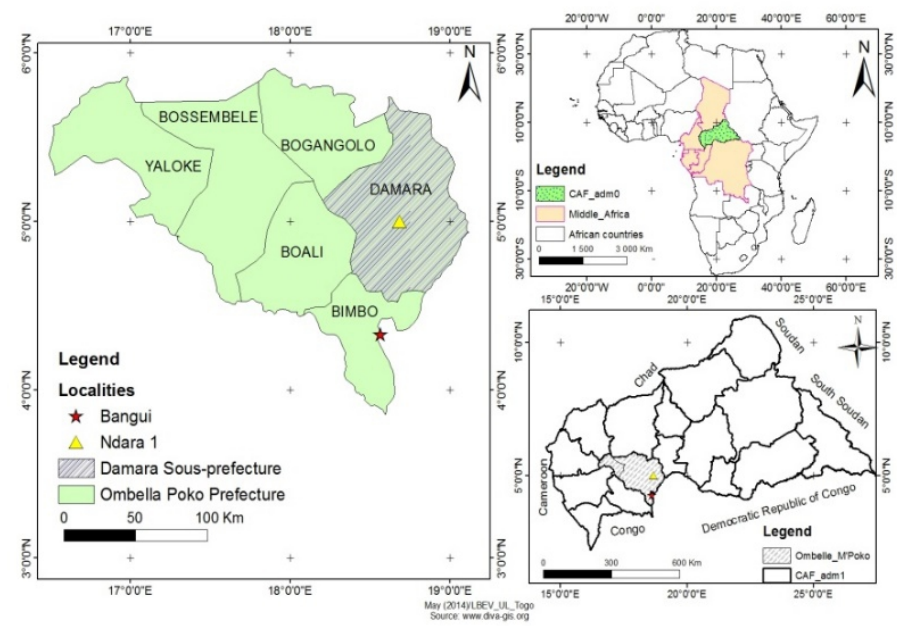

Figure 1 : Zone d'étude

\section{Matériel végétal}

Le matériel végétal a été issu d'un cultivar de manioc “Gabon”. Ce cultivar a été choisi par rapport aux résultats des travaux antérieurs réalisés par l'équipe des chercheurs du Laboratoire des Sciences Biologiques et Agronomiques pour le Développement (LaSBAD) de la Faculté des Sciences à l'Université de Bangui (Centrafrique). Il a été identifié comme cultivar résistant à la mosaïque africaine de manioc (CMD), performant du point de vue rendement, très apprécié par les producteurs du manioc et disponible dans le site pilote d'étude (Zinga et al., 2012). Des boutures de manioc ont été constituées par 2020 micro-boutures de manioc d'écotype Gabon agées de 9 à 15 mois.

\section{Matériel fertilisant}

Le matériel fertilisant a été constitué: i) d'une source d'azote : urée $\left(\mathrm{Co}\left(\mathrm{NH}_{2}\right)_{2}\right.$ contenant $46,65 \%$ de $\mathrm{N}$, ii) de deux sources de potassium : le Sulphate de potassium $\left(\mathrm{K}_{2} \mathrm{SO}_{4}\right)$ contenant $44,8 \%$ de $\mathrm{K}$, et le chlorure de potassium (KCI) contenant 52,4 \% de $\mathrm{K}$, iii) d'une source de $\mathrm{P}$ : le triple super phosphate TSP $\left(\mathrm{Ca}\left(\mathrm{H}_{2} \mathrm{PO}_{4}\right)_{2}\right.$ contenant $26,46 \%$ de $\mathrm{P}$. Les doses appliquées pour ces qutre types de fertilisant ont été de 0, 40, 120 et $200 \mathrm{~kg}$. $\mathrm{ha}^{-1}$.

\section{Méthodologie}

\section{Collecte des données}

L’étude a porté sur le prélèvement et l'analyse physico-chimique des échantillons de sol et sur le suivi agronomique de parcelles expérimentales 
pendant douze (12) mois. Les tubercules issus de l'essai ont été dégustés pour apprécier leur qualité organoleptique.

\section{Prélèvement d'échantillons de sol}

Des échantillons de sol ont été prélevés avant l'apport de fertilisant au moyen d'une tarière manuelle dans les vingt premiers centimètres supérieurs assimilables à l'horizon organique $(0$ à $20 \mathrm{~cm})$. Les sols ont été prélevés par randomisation. Les échantillons ont été prélevés respectivement au centre et aux quatre coins du champ de 0,75 ha et ont été mélangés pour constituer un échantillon composite de $1 \mathrm{~kg}$ par point de prélevement (Buol et al., 2011). Ces échantillons composites ont été séchés à la température ambiante de laboratoire $\left(25^{\circ}\right.$ à $\left.30^{\circ} \mathrm{C}\right)$ avant la séparation des éléments grossiers de la terre fine au moyen d'un tamis de $2 \mathrm{~mm}$. Les échantillons de terre fine ont été conditionnés dans des sachets plastiques et transmis au laboratoire d'analyse des eaux, sols et végétaux du CIRAD de MontpellierFrance, pour des analyses physico-chimiques. Les résultats analytiques de sol ont été comparés à des valeurs seuils des normes de références.

\section{Analyses physico-chimiques d'échantillons de sol}

L’analyse physique en cinq fractions granulométriques (argile, limons fins et grossiers, sables fins et grossiers) a été réalisée par la méthode de pipette Robinson suivant la norme AFNOR NF X31-107 (Buol et al., 2011). L’analyse du carbone et de l'azote total a été effectuée par les méthodes décrites dans la norme internationale NF ISO 10694 pour le carbone et NF ISO 13878 pour l'azote (Buol et al., 2011). Les pH eau et KCl ont été déterminés suivant la norme internationale NF ISO 10390 (Moufti and Mountadar, 2004). La capacité d'échange cationique (Saragoni et al., 1992) a été mesurée par la méthode Metson selon la norme AFNOR NF X31-130 (Buol et al., 2011). La teneur en éléments $\mathrm{Al}^{3+}, \mathrm{Ca}^{2+}, \mathrm{Cu}^{2+}, \mathrm{K}^{+}$, $\mathrm{Mg}^{2+}, \mathrm{Mn}^{2+}, \mathrm{Na}^{+}, \mathrm{Zn}^{2+}$ a été déterminée par la méthode fluoro-nitroperchlorique. Le phosphore total et le phosphore assimilable ont été dosés suivant la norme internationale NF ISO 11263 (Buol et al., 2011).

\section{Mise en culture}

Des micro-boutures de 25 à 30 cm de la variété de manioc Gabon comportant 5 à 8 nœuds ont été repiquées pour la mise en place du dispositif expérimental de l'essai. Le dispositif mise en place et adopté est celui de la randomisation complète avec 4 blocs (4 répétitions) et de 2 facteurs étudiés. Les engrais minéraux (Urée, Sulfate de potassium, Chlorure de potassium et Triple superphosphate) constituent le facteur principal avec quatre doses de traitements, à savoir : le témoin sans engrais 0, les doses 40, 120 et $200 \mathrm{Kg}$ $\mathrm{ha}^{-1}$, soit six (6) niveaux de traitements qui sont représentés dans le tableau 1. 
Le cultivar du manioc Gabon, représente un second facteur avec 14 traitements (13 traités et 1 témoin non traité) de 56 parcelles élémentaires. Chaque traitement représente une parcelle élémentaire d'une superficie de $36 \mathrm{~m}^{2}$ soit $6 \mathrm{~m} \times 6 \mathrm{~m}$. L'espacement entre les pieds de manioc est de $1 \mathrm{~m}^{2}$ et 2 $\mathrm{m}$ entre les parcelles élémentaires puis $3 \mathrm{~m}$ entre les blocs. Un bloc est constitué de $14 \mathrm{~m}$ de largeur et de $54 \mathrm{~m}$ de longueur, qui est subdivisé en 14 parcelles élementaires. Ainsi, la surface cultivable par bloc a été de $14 \mathrm{~m} \mathrm{x}$ $54 \mathrm{~m}$, soit $756 \mathrm{~m}^{2}$, et d' une surface totale de culture sur les qutre blocs de $3024 \mathrm{~m}^{2}$.

Tableau 1 : Combinaison des doses d'engrais minéraux utilisées

\begin{tabular}{|c|c|c|c|c|c|}
\hline \multirow[b]{2}{*}{ Série } & \multirow[b]{2}{*}{ Traitements } & \multicolumn{4}{|c|}{ Engrais minéraux } \\
\hline & & $\begin{array}{c}\text { Urée } \\
\text { Kg ha }^{-1}\end{array}$ & $\begin{array}{l}\mathrm{K}_{2} \mathrm{SO}_{4}, \\
\mathrm{Kg} \mathrm{ha}^{-1}\end{array}$ & $\begin{array}{c}\mathrm{KCl} \\
\mathrm{Kg} \mathrm{ha}^{-1} \\
\end{array}$ & $\begin{array}{c}\text { TSP } \\
\text { Kg ha }^{-1} \\
\end{array}$ \\
\hline $\begin{array}{c}1 \\
\text { Effet contrôle } \\
\end{array}$ & $\mathrm{T}_{0}$ & 0 & 0 & 0 & 0 \\
\hline $\begin{array}{c}2 \\
\text { Effet P }\end{array}$ & $\mathrm{T}_{1}$ & 0 & 0 & 0 & 40 \\
\hline \multirow{3}{*}{$\begin{array}{c}3 \\
\text { Effet } \mathrm{N} \text { et } \mathrm{P}\end{array}$} & $\mathrm{T}_{2}$ & 40 & 0 & 0 & 40 \\
\hline & $\mathrm{T}_{3}$ & 120 & 0 & 0 & 40 \\
\hline & $\mathrm{T}_{4}$ & 200 & 0 & 0 & 40 \\
\hline \multirow{3}{*}{$\begin{array}{c}4 \\
\text { Effet } \mathrm{K} \text { et } \mathrm{P}\end{array}$} & $\mathrm{T}_{5}$ & 0 & 40 & 0 & 40 \\
\hline & $\mathrm{T}_{6}$ & 0 & 120 & 0 & 40 \\
\hline & $\mathrm{T}_{7}$ & 0 & 200 & 0 & 40 \\
\hline \multirow{3}{*}{$\begin{array}{c}5 \\
\text { Effet N, P et K }\end{array}$} & $\mathrm{T}_{8}$ & 40 & 40 & 0 & 40 \\
\hline & $\mathrm{T}_{9}$ & 120 & 120 & 0 & 40 \\
\hline & $\mathrm{T}_{10}$ & 200 & 0 & 0 & 40 \\
\hline \multirow{3}{*}{$\begin{array}{c}6 \\
\text { Effet } \mathrm{K}^{*} \text { et } \mathrm{P}\end{array}$} & $\mathrm{T}_{11}$ & 0 & 0 & 40 & 40 \\
\hline & $\mathrm{T}_{12}$ & 0 & 0 & 120 & 40 \\
\hline & $\mathrm{T}_{13}$ & 0 & 0 & 200 & 40 \\
\hline
\end{tabular}

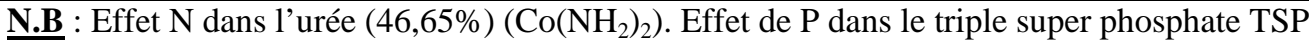
$(26,46 \%)$ ou $\left(\mathrm{Ca}\left(\mathrm{H}_{2} \mathrm{PO}_{4}\right)_{2}\right)$. Effet de $\mathrm{K}$ dans le Sulfate de potassium $(44,8 \%)\left(\mathrm{K}_{2} \mathrm{SO}_{4}\right)$. Effet de

$\mathrm{K}$ dans le chlorure de potassium $(52,4 \%) \mathrm{KCl}$. $\mathrm{K}^{*}$ correspond au $\mathrm{K}$ provenant de $\mathrm{KCl}$.

Il est indiqué dans ce tableau la quantité des combinaisons d'engrais à fournir au sol, au pied des jeunes plantules de manioc après un mois de plantation. Les engrais sont pesés et conditionnés dans un sac à conditionnement en fonction des traitements.

\section{Mode d' application des fertilisants minéraux}

Au cours de cette étude, nous avons utilisé la méthode de la localisation en ligne pour appliquer les engrais, en utilisant la méthode de «split-application», à partir d'un mois après le bouturage. Cette méthode consiste à diviser la quantité d'intrant en plusieurs fois en fonction du cycle végétatif du manioc et des traitements retenus pour l'expériementation. 
Dans l'étude, l'urée et le sulfate de potassium ont été appliqués en trois fois et le phosphore en une seule application. D’abord, 1/3 de la quantité d'urée et de potassium a été appliquée après un mois de la plantation (après reprise de la bouture), ensuite $1 / 3$ à 3 mois et enfin le dernier tiers au $5^{\text {ieme }}$ mois de la plantation. Quand au phosphore, c'est sa quantité totale qui a été appliquée lors du premier traitement. La quantité de fertilisant par pied/traitement est donnée au Tableau 1. La technique d'épandage localisée des engrais a été appliquée à $5 \mathrm{~cm}$ des boutures de manioc repiquées. Elle était faite en traçant un cercle creux de $5 \mathrm{~cm}$ autour du pied de manioc. Après 12 mois d'essai, les tubercules du manioc ont été récoltés pour évaluer le rendement moyen en poids frais et en nombre de tubercules par plant selon les traitements (doses d'engrais minéraux apportées).

\section{Test de dégustation de tubercule cru et de boule manioc}

L'échantillonnage a pris en compte les producteurs et les étudiants. L'enquête a été réalisée en focus groupe en tenant compte des différentes composantes sociales pouvant être capables de donner une bonne appréciation des tubercules ou de la boule de manioc à déguster. Le choix des enquêtés a été réalisé de façon aléatoire sans distinction de sexe. La méthode d'entretin directif comportant une serie des questions semiouvertes, qui a été adopté lors de cette enquête et sur les paramètres organolepetiques d'appéciation de boule de manioc suivant : la consistance, la plasticité, l'odeur et le goût. Pour l'appréciation de la qualité organoleptique des tubercules, les paramètres choisis ont été : l'amertume et la teneur en eau lors de la dégustation de tubercule cru et après la mastication.

Cette méthode a permi de vérifier les raisons qui ont poussées les répondants à donner leur avis sur les différents paramètres choisis; mais aussi de vérifier l'hypothèse selon laquelle, certaines variétés améliorées du manioc, introduites et locales; que les tubercules n'ont pas une bonne teneur en eau; de même que la boule de manioc après sa prépartion n'est pas consistante et est très plastique (colle les doigts), si elle est laissée à l'air libre pendant un certain temps.

Après la récolte, des tubercules crus ont été soignement lavés avec de l'eau saine, puis découpés en petits morceaux et étiquetés par traitement pour faciliter le test de dégustation.

Pour les boules de manioc, les tubercules ont été épluchés, puis trempés dans de l'eau propre (rouissage) pendant trois jours, puis égouttés et du pressés dans un sac pendant un jour, et enfin séchés au soleil. Après le séchage, les cossettes récupérées ont été écrasées au moulin à manioc. La farine obtenue est utilisée pour préparer les boules de manioc. 
Cinq marmites ont été mises au feu contenant chacun un litre d'eau. Ensuite, à l'aide de la balance mécanique, $500 \mathrm{~g}$ de la farine de manioc sont ajoutés à l'eau bouillante, puis le mélange (malaxé) avec un baton pour obtenir au bout de 5 minutes une boule de manioc prête à consommer.

Chaque personne enquêté passe à tour de rôle pour faire le test. La bonne appréciation est notée oui $=1$ et la mauvaise appréciation est notée non $=0$. Ces notations ont permis d'orienter lesanalyses et les interprétations des résultats des données d'enquête.

\section{Traitement des données}

Les données collectées sur le terrain et les résultats d'analyse ont été saisis et codifiés à l'aide du tableur Microsoft Excel 2010 ${ }^{\circledR}$. Cet ensemble a été soumis à des analyses statistiques en utilisant le logiciel $\mathrm{R}$ version 3.1.2 (2014-10-31) et le tableur Excel ${ }^{\circledR}$. Le test HSD de Turkey «Honestly Significant difference ", a permis d'évaluer la significativité des différences de rendement en poids et en nombre des tubercules frais des plants de manioc selon les apports de fertilisants. Le test de Dunnett à comparaison multiple entre le traitement témoin et les autres traitements, a été utilisé pour analyser les effets individuels sur les rendements des apports d'azote $(\mathrm{N})$, de phosphore $(\mathrm{P})$ et de potassium $(\mathrm{K})$ des engrais utilisés.

\section{Résultats}

\section{Caractéristiques physico-chimiques des échantillons de sols prélevés avant l'apport de fertilisant}

15 répétitions ont permis d’obtenir les résultats présentés dans les tableaux 2 et 3. L'analyse granulométrique montre que le sol de la zone d'étude est de type loameux avec $20 \%$ d' argile, $30 \%$ de limon et $50 \%$ de sable.

Tableau 2: Analyse granulométrique des échantillons de sols étudiés (horizon : 0-20 cm)

\begin{tabular}{|c|c|}
\hline Eléments & Teneur (\%) \\
\hline Argiles & 20 \\
\hline Limons & 30 \\
\hline Sables & 50 \\
\hline
\end{tabular}

Ainsi que le montre le tableau 3, la moyenne de $\mathrm{pH}$ des échantillons de sol est de 5,7. Le sol est très pauvre en matières organiques et en azote total par rapport à la norme de référence d'interprétation d'analyse du sol (Buol et al., 2011). La teneur en carbone organique est moyenne (1,28 \%). Le rapport $\mathrm{C} / \mathrm{N}$ des échantillons de sols étudiés se situe dans la norme avec une moyenne de 14,20. La teneur moyenne en phosphore assimilable est de $3,01 \mathrm{mg} \mathrm{Kg}^{-1}$ et se situe dans l'intervalle de seuil critique (3 à $8 \mathrm{mg} \mathrm{Kg}^{-1}$ ). Le potassium total a une valeur moyenne de $0,22 \mathrm{~g} \mathrm{~kg}^{-1}$ et se situe du seuil 
critique 0,20-0,23 g. $\mathrm{kg}^{-1}$. La somme des cations échangeables $\left(\mathrm{Ca}^{2+}, \mathrm{Mg}^{2+}\right.$, $\mathrm{K}^{+}$et $\mathrm{Na}^{+}$) et la capacité d'échange cationique (CEC) des échantillons de sol analysés présentent des valeurs très inférieures aux normes de référence d'interprétation d'analyse du sol. Les taux de saturation (V) en bases échangeables des sols présentent une moyenne de $85 \%$ et se situent dans l'intervalle des valeurs de référence 60 - 90 \% (Tableau 3).

Tableau 3: Analyse chimique des sols étudiés (horizon : 0-20 cm)

\begin{tabular}{|c|c|c|}
\hline Eléments & Teneur & Norme* $^{*}$ \\
\hline pH eau & 5,7 & 4,5 \\
\hline pH KCl & 4,6 & $3,6-5,5$ \\
\hline Matière organique (\%) & 2 & $1,26-2,5$ \\
\hline Carbone organique (\%) & 1,28 & $0,12-0,22$ \\
\hline Azote total (\%) & 0,07 & $9-15$ \\
\hline Rapport C/N & 14,2 & $3-8$ \\
\hline P assimilable (mg Kg ${ }^{-1}$ ) & 3,01 & $0,20-0,23$ \\
\hline P total (g Kg-1) & 0,22 & 3,7 \\
\hline K (g Kg-1) & 5,4 & $5-8$ \\
\hline Ca éch (méq 100g-1) & 2,5 & $1,5-3$ \\
\hline Mg éch (méq 100g-1) & 0,78 & $0,15-0,25$ \\
\hline K éch (méq 100g-1) & 0,12 & $0,3-0,7$ \\
\hline Na éch (méq 100g-1) & 0,04 & - \\
\hline Al éch (méq 100g-1) & 0,08 & $0,01-0,10$ \\
\hline Mn éch (méq 100g-1) & 0,27 & - \\
\hline H éch (méq 100g-1) & 0,04 & $7,5-15$ \\
\hline S(Ca, Mg, K, Na) (méq 100g-1) & 3,05 & $10 \leq \mathrm{CEC} \leq 20$ \\
\hline CEC (méq 100g-1) & 3,4 & $60 \leq \mathrm{TS}<90$ \\
\hline V (\%) & 85,07 & $1996)$ \\
\hline
\end{tabular}

*pH eau de référence pour la culture du manioc (Buol et al., 2011) ; (Howeler, 1996)

*Valeurs normatives de référence (Doucet, 2006; Giroux and Audesse, 2004; Howeler, 1996, 2001)

\section{Effet des fumures sur le rendement en poids et en nombre des tubercules frais de manioc}

Le rendement en poids moyen de tubercules sur sol traité avec les engrais minéraux NPK dans la zone d'étude est de 25 t.ha ${ }^{-1}$. Le meilleur rendement obtenu à l'issue de cette expérimentation est le traitement $\mathrm{T}_{10}$ (200 kg/ha d'azote (N) $40 \mathrm{~kg} /$ ha phosphore (P) et $200 \mathrm{~kg} / \mathrm{ha}$ de potassium $\left(\mathrm{K}-\mathrm{K}_{2} \mathrm{SO}_{4}\right)$ avec un rendement moyen de 40,20 t.ha ${ }^{-1}$ et un taux d'accroissement de $115 \%$ par rapport au témoin non traité $\mathrm{T}_{0}$ qui a un rendement moyen de 18,70 t.ha ${ }^{-1}$ et un taux d'accroissement nul (Figure 7 B). Le rendement le plus faible (12 t.ha ${ }^{-1}$ en moyenne) est le traitement $T_{13}$. Le test HSD de Tukey, a montré qu'il y a une différence significative de rendement en poids frais des tubercules de manioc entre les traitements $T_{10}$, $\mathrm{T}_{13}, \mathrm{~T}_{12}, \mathrm{~T}_{1}$ et $\mathrm{T}_{0}$ (Figure $7 \mathrm{~B}$ et Tableau 4). Comme pour le rendement en poids moyen de tubercules, le rendement en nombre de tubercules le plus élevé est celui du traitement $T_{10}$ avec 6,67 tubercules.plant ${ }^{-1}$. Le témoin non traité fournit 5 tubercules.plant ${ }^{-1}$ en moyenne. Le plus faible rendement en 
nombre est le traitement $\mathrm{T}_{13}(0 \mathrm{Kg} /$ ha d'azote $(\mathrm{N}) 40 \mathrm{Kg} /$ ha phosphore (P) et $200 \mathrm{Kg} /$ ha de potassium $(\mathrm{K}-\mathrm{KCl})$, qui a 3,25 tubercules plant ${ }^{-1}$ en moyenne. Le test HSD de Tukey, a montré qu'il y a une différence très significative du rendement en nombre des tubercules de manioc entre le traitement $\mathrm{T}_{10}$ (dose maximale d'engrais) et les traitements sans apport d'azote $T_{13}, T_{12}, T_{1}$ et $T_{0}$. On remarque aussi que les rendements les plus élevés correspondent aux traitements $\mathrm{T}_{8}, \mathrm{~T}_{9}$ et $\mathrm{T}_{10}$ avec des apports simultanés de $\mathrm{N}$, $\mathrm{K}$ et $\mathrm{P}$ (Figure 7 A).
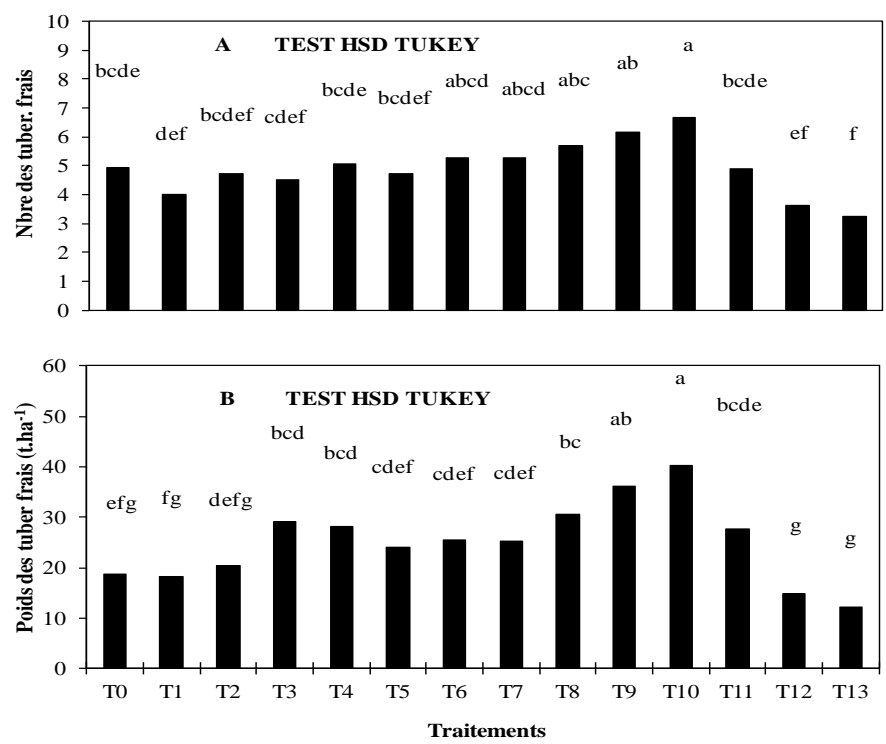

Test HSD (Honestly Significant Difference) de Tukey:alpha: 0.05 ; Df Error: 546 Critical Value of Studentized Range: 4.76. HSD : 0.922 Means with the same letter are not significantly different

Figure 7 : Rendement moyen des tubercules frais du manioc en fonction des traitements

Tableau 4: Taux d'accroissement des tubercules frais de manioc

\begin{tabular}{ccccc}
\hline Traitements & $\begin{array}{c}\text { Nombre } \\
\text { tubercules/plan }\end{array}$ & Taux d'accrois. (\%) & Poids $\left(\mathrm{t} \mathrm{ha}^{-1}\right)$ & Taux d'accrois. (\%) \\
\hline T0 & 5 & 0 & 18,7 & 0 \\
T1 & 4 & -20 & 18,1 & $-3,2$ \\
T2 & 4,7 & -6 & 20,2 & 8,02 \\
T3 & 4,5 & -10 & 29,1 & 55,62 \\
T4 & 5,05 & 10 & 28,1 & 50,26 \\
T5 & 4,7 & -6 & 24 & 28,34 \\
T6 & 5,25 & 5 & 25,4 & 35,83 \\
T7 & 5,25 & 5 & 25,2 & 34,76 \\
T8 & 5,67 & 13,4 & 30,6 & 63,64 \\
T9 & 6,15 & 23 & 36,1 & 93,05 \\
T10 & 6,67 & 33,4 & 40,2 & 114,97 \\
T11 & 4,87 & $-2,6$ & 27,5 & 45,06 \\
T12 & 3,6 & -28 & 14,8 & $-20,85$ \\
T13 & 3,25 & -35 & 12 & $-35,83$ \\
Moyenne & 5 & & 25 & \\
\hline
\end{tabular}




\section{Effet des fumures sur la croissance et le rendement du manioc}

La croissance des plants de manioc du cultivar local Gabon est normale avec une hauteur moyenne de 2,06 $\pm 0,50 \mathrm{~m}$. La croissance moyenne en diamètre des plants est de $3,50 \pm 0,87 \mathrm{~cm}$. Le traitement $\mathrm{T}_{10} \mathrm{a}$ mieux réagi au traitement avec la fumure minérale sur la croissance en hauteur et diamètre moyens des plants, ainsi que sur le rendement en poids et en nombre des tubercules frais de manioc, par rapport aux traiments $\mathrm{T}_{13}$ et $\mathrm{T}_{0}$ (Figure 8).

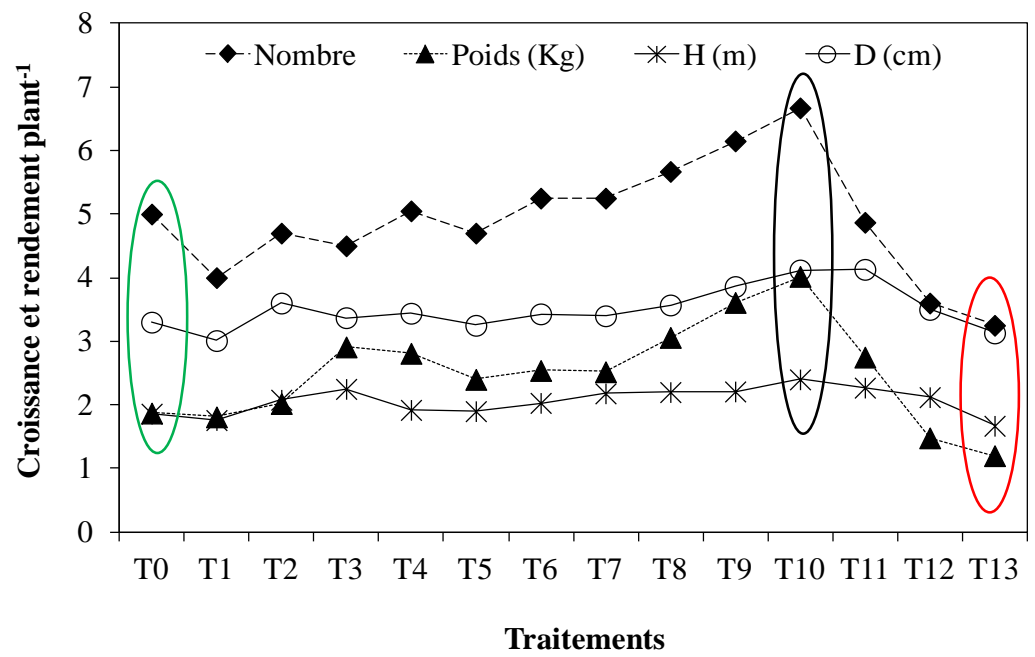

Figure 8: Rendement des tubercules frais du manioc en fonction des traitements

\section{Effet des apports de $\mathbf{N}, \mathbf{P}$ et $\mathrm{K}$ sur le rendement}

Les résultats cette étude ont montré qu'il y a les effets d'approts de $\mathrm{N}$ et $\mathrm{P}\left(\mathrm{T}_{3}\right.$ et $\left.\mathrm{T}_{4}\right)$; il y a plus d'effets d'approts de $\mathrm{N}, \mathrm{P}$ et $\mathrm{K}\left(\mathrm{T}_{8}, \mathrm{~T}_{9}\right.$ et $\left.\mathrm{T}_{10}\right)$ sur rendement du manioc au cours de cette étude. Par contre pas d'effet d'apports de $K$ et $P$ sur le rendement du manioc $\left(T_{5}, T_{6}, T_{7}, T_{12}\right.$ et $T_{13}$, sauf $\mathrm{T}_{11}$ ) (Figure 8, Tableau 4).

L'analyse statistique à travers le test de Dunnett a montré qu'il y a une très différence significative avec une valeur de $\mathrm{P}$ value $(\mathrm{P}<0.01)$, au seuil (alpha $=0,005)$ sur l'apport de potassium $(\mathrm{K})$ en provenance de Sulfate de potassium $\left(\mathrm{K}_{2} \mathrm{SO}_{4}\right)$ et l'apport de l'azote en provenance d'urée sur les traitements $T_{3}, T_{4}, T_{8}, T_{9}$ et $T_{10}$ par rapport au témoin non traité $T_{0}$. Par contre, pas de différence entre le témoin et les autres traitements $\left(T_{1}, T_{2}, T_{5}\right.$, $\mathrm{T}_{6}, \mathrm{~T}_{7}, \mathrm{~T}_{12}$ et $\mathrm{T}_{13}$, sauf $\mathrm{T}_{11}$ l'apport de $\mathrm{P}$ est constant pour tous les traitements sauf le témoin $\mathrm{T}_{0}$ (Tableau 5). 
Tableau 5: Test de comparaison multiple de Dunnet effectué sur le rendement en poids frais de tubercule de manioc

\begin{tabular}{|c|c|c|c|}
\hline Comparaison & Différe & nce q & value \\
\hline T0 vs T1 & 0.05950 & $0.2178 \mathrm{~ns}$ & $\mathrm{P}>0.05$ \\
\hline T0 vs T2 & -0.1575 & $0.5766 \mathrm{~ns}$ & $\mathrm{P}>0.05$ \\
\hline T0 vs T3 & -1.043 & $3.817 * *$ & $\mathrm{P}<0.01$ \\
\hline T0 vs T4 & -0.9465 & $3.465 * *$ & $\mathrm{P}<0.01$ \\
\hline T0 vs T5 & -0.5388 & $1.972 \mathrm{~ns}$ & $\mathrm{P}>0.05$ \\
\hline T0 vs T6 & -0.6715 & $2.458 \mathrm{~ns}$ & $\mathrm{P}>0.05$ \\
\hline T0 vs T7 & -0.6453 & $2.362 \mathrm{~ns}$ & $\mathrm{P}>0.05$ \\
\hline T0 vs T8 & -1.196 & $4.377 * *$ & $\mathrm{P}<0.01$ \\
\hline T0 vs T9 & -1.743 & $6.380 * *$ & $\mathrm{P}<0.01$ \\
\hline T0 vs T10 & -2.154 & $7.886 * *$ & $\mathrm{P}<0.01$ \\
\hline T0 vs T11 & -0.8860 & $3.244 *$ & $\mathrm{P}<0.05$ \\
\hline T0 vs T12 & 0.3910 & $1.431 \mathrm{~ns}$ & $\mathrm{P}>0.05$ \\
\hline T0 vs T13 & 0.6623 & $2.424 \mathrm{~ns}$ & $\mathrm{P}>0.05$ \\
\hline
\end{tabular}

The $\mathrm{P}$ value is $<0.0001$, considered extremely significant.

Variation among column means is significantly greater than expected by chance.

Control column: T0. If the value of $q$ is greater than 2.790 then the $P$ value is less than 0.05 .

\section{Caractéristiques organoletique des tubercules crus et des boules de manioc}

\section{Profil des dégustateurs durant l'enquête}

$\mathrm{Au}$ total 85 personnes ont participé au test de dégustation des tubercules crus de manioc. Les femmes occupent une proportion de $61 \%$ contre $39 \%$ d' hommes (Figure 9A).

L’appréciation de la qualité organoleptique des boules de manioc a mobilisé 135 dégustateurs au total, dont $67 \%$ de femmeset 33\% hommes (Figure $9 \mathrm{~B}$ ). Pour les types de dégustateurs la tranche d'âge la plus représentée est la tranche25-45 ans (Figure 9A \& B).

\section{Pourcentage de participation à l'enquête des étudiants et des producteurs}

Pour l'apprécier la qualité organoleptique des boules de manioc en fonction du type de fertilisation,l'enquête a été menée auprès des paysans producteurs et d'universitaires.

L'échantillon de 135 personnes intérrogées comprend 90 (67\%) producteurs et 34 (33\%) universitaires ont donné leur avis sur l'appréciation de la qualité des boules du manioc traité avec les engrais minéraux. La 
tranche d’âge moyenne est comprise entre 25 et 45 ans et il occupe une proportion de 46,67\% au cours de cette étude (Figure 10).

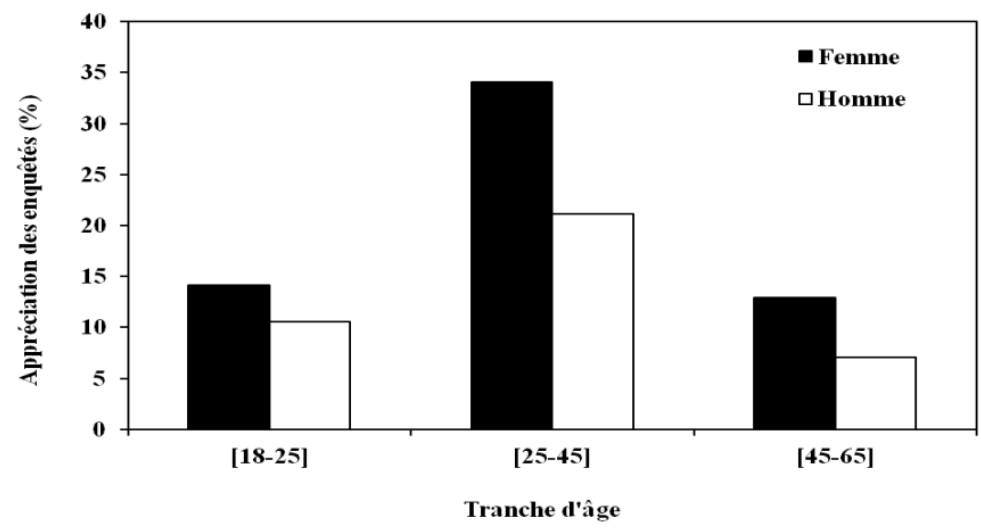

Figure 9A: Répartition des dégustateurs de tubercules crus de manioc selon l’âge et le sexe

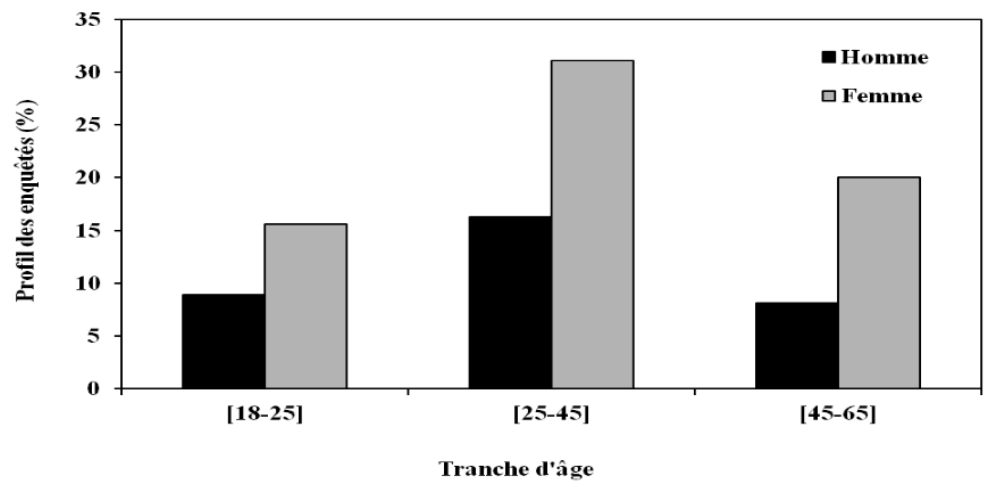

Figure 9 B : Répartition des dégustateurs de la boule de manioc selon l’âge et le sexe

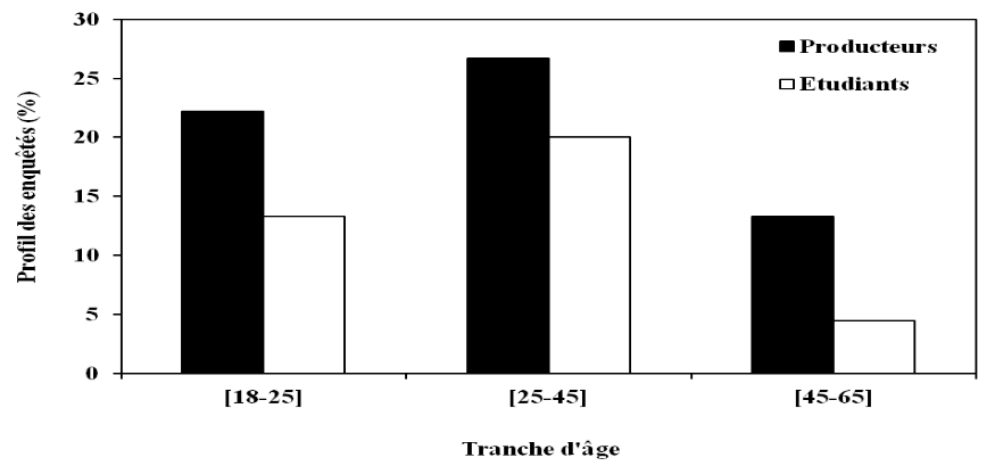

Figure 10 : Taux de participation des étudiants et producteurs du manioc à l'enquête

\section{Teneur en eau et gout des tubercules crus de manioc}

Le test utilisé pour ce type d'analyse consiste à dire si oui ou non, il y a une bonne teneur en eau. C'est ainsi que, $91 \%$ des dégustateurs ont rapporté que le traitement témoin $\left(\mathrm{T}_{0}\right)$ présente une bonne teneur en eau. Le traitement $\mathrm{T}_{11}$ a recueilli $94 \%$ de réponse positive d'appréciation de la teneur 
en eau. Par contre le traitement $\mathrm{T}_{9}$ a recueilli le plus faible taux d’appréciation positive avec 76 \% (Figure 9C).

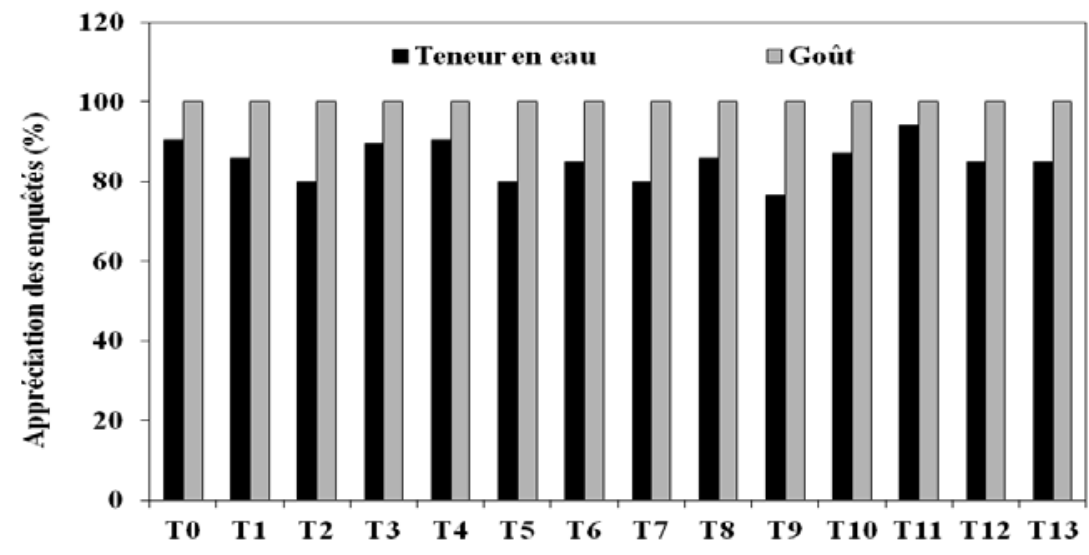

Figure 9C : Pourcentage d'évaluation de la teneur en eau et du gout du manioc d'après les enquêtés

\section{Appréciation de la qualité organoleptique de la boule du manioc}

Les résultats d'enquête concernent les paramètres organoleptiques suivants : consistance, plasticité, odeur et goût.

Selon les réponses des dégustation des boules à l'issu de cette enquête, ils ont répondu qu'il n’y a pas d'effet des fumures minérales sur la qualité de boule de manioc appréciée par les enquêtés.

\section{Consistance et plasticité de la boule de manioc}

Les dégustateurs ont appréciés à $82 \%$ en moyenne sur tous les traitements, que les boules préparées sont moyennement consistantes et facile à couper avec les doigts. Le traitement $T_{1}$ a rapporté une très bonne proportion d'appréciation de la consistance de la boule par rapport aux autres traitements avec $82 \%$, suivi du traitement $\mathrm{T}_{2}$ et $\mathrm{T}_{11}$ qui ont reccuilli chacun $79 \%$. Par contre le traitement $\mathrm{T}_{8}$, a receuilli le plus faible taux d'appréciation de la consistance de boule de manioc.

Quant à l'appréciation de la plasticité de boule, le traitement $\mathrm{T}_{2}$ a rapporté $74 \%$ de proportion d'appréciation positive, selon les avis des répondants. Le traitement $\mathrm{T}_{11}$ a recueilli $68 \%$ et c'est la plus faible proportion d'appréciation rapporté par les répondants au cours de cette enquête (Figure 11A). 


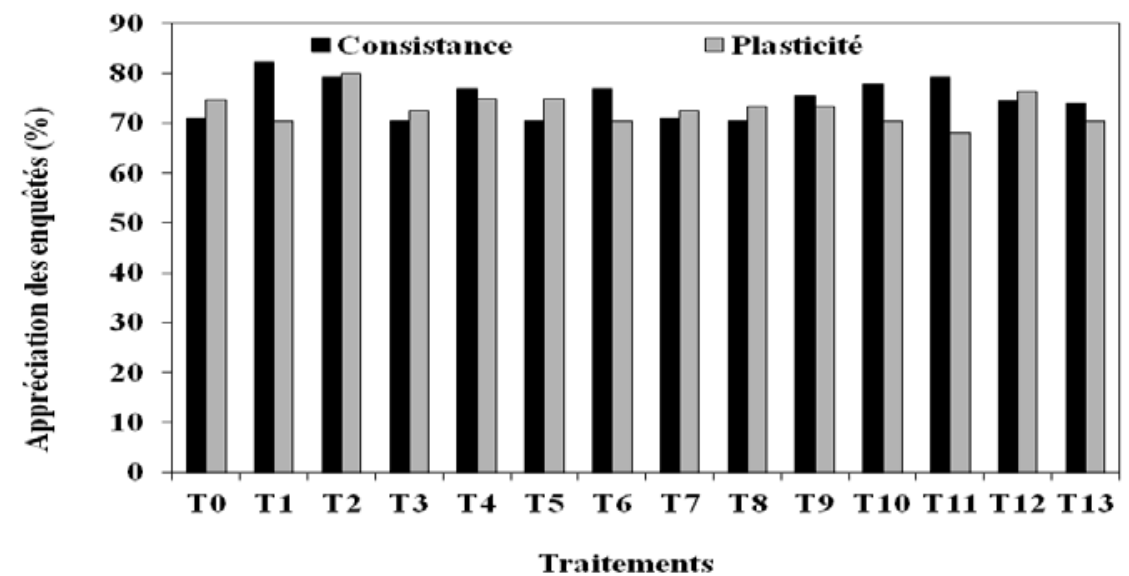

Figure 11A : Pourcentage d’appréciation de la consistance et de la plasticité de la boule de manioc d'après les enquêtés

\section{Odeur et goût de la boule de manioc}

Les résultats de cette étude ont montré qu'il n'y a pas d'effet d'odeur et de goût des engrais minéraux sur la boule de manioc du témoin $\left(\mathrm{T}_{0}\right)$ et des autres traitements, selon les avis des répondants. L'appréciation moyenne de l'odeur et de goût de la boule de manioc, d'après les répondants sont respectvement de 81 et $80 \%$. Les traitements $\mathrm{T}_{1}$ et $\mathrm{T}_{6}$ ont recueilli les fortes proportions sur l'appréciation de l'odeur avec 89 \% chacun. Ensuite, le traitement $\mathrm{T}_{10}$ qui a remporté $90 \%$ de forte proportion sur l'appréciation sur de goût de la boule de manioc au cours de cette enquête. Le traitement $T_{9}$, a recueilli la plus faible proportion avec respectivement $64 \%$ et $69 \%$ sur l'appréciation de l'odeur et de goût de la boule de manioc selon les dégustateurs (Figure 11B).

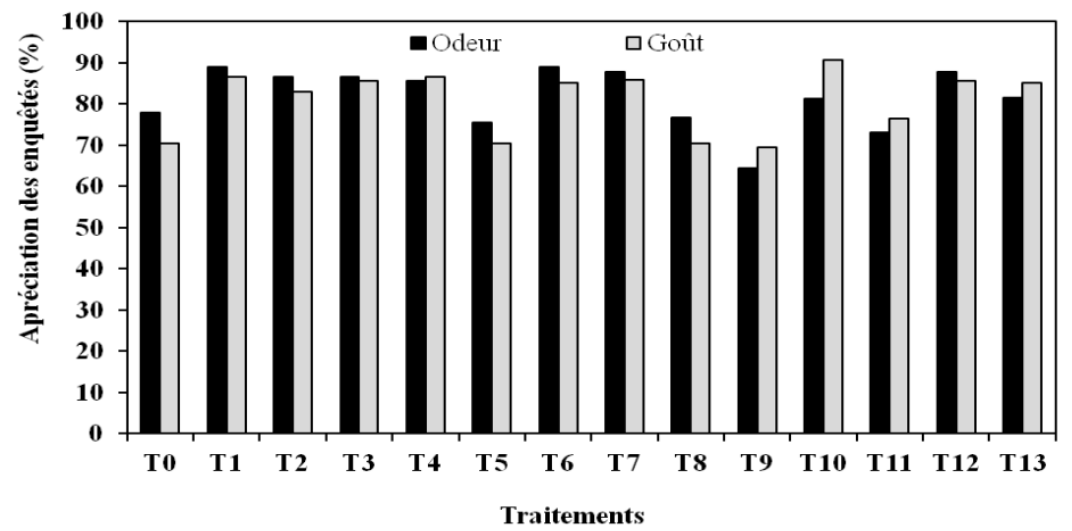

Figure 11B: Pourcentage d'appréciation de l’odeur et du goût de la boule de manioc 


\section{Discussion}

Les résultats granulométriques montrent que plus de $93 \%$ des échantillons de sols ont une texture loameuse c'est-à-dire une texture moyenne de type terre franche. Ces résultats se rapprochent de ceux de Giguère et al,.(2002) et de Pypers et al,.(2011) (Giguère, 2002), (Pypers et al., 2011). La moyenne de $\mathrm{pH}$ eau de sol étudié est de 5,7 et est moyennement acide (Staff, 1993). La teneur en azote total est très faible et par conséquent la minéralisation de la matière organique est très faible. Le sol est très pauvre en matières organiques de même que la teneur en azote total par rapport à norme de référence d'interprétation d'analyse du sol (Akanza et al., 2002). Le rapport C/N des échantillons de sol étudiés (14.2) ne se situe pas au delà de seuils critiques. La somme des cations échangeables $\left(\mathrm{Ca}^{2+}, \mathrm{Mg}^{2+}, \mathrm{K}^{+}\right.$et $\left.\mathrm{Na}^{+}\right)$est également très faible avec une valeur de 3,05 méq $100 \mathrm{~g}^{-1}$ (Saragoni et al., 1992). Le taux de saturation en bases échangeables des échantillons de sols étudié est de 85\%. Cette valeur se situe dans l'intervalle par rapport au seuil critique qui varie de $60-90 \%$. Ces résultats sont similaires à ceux obtenus par Giroux et La france (Giroux and Audesse (2004); La France et al. (2012)).

Dans cette étude, le rendement du manioc, en poids frais de tubercules à l'hectare, a varié de 18.7 t.ha ${ }^{-1}$ pour le traitement témoin sans engrais $\left(T_{0}\right)$, à 40,2 t.ha ${ }^{-1}$ pour le meilleur traitement $\left(T_{10}\right)$ avec un taux d'accroissement non négligeable de 114,94 \% (cf. Tableau 4). Les valeurs obtenues dans cette étude, sont du même ordre de grandeur que celles rapportées par Zinga et al. (2013), qui ont obtenu sans apport minéral au sol, dans la même zone d'étude et pour la même variété locale "Gabon', un rendement moyen en poids frais de tubercule de 18,01 t.ha' ${ }^{-1}$ (Zinga et al., 2013). Ces valeurs sont également de l'ordre de grandeur des rendements de 20 t.ha $^{-1}$ obtenus par Howeler (1985 et 2011) au nord du Vietnam, après toutefois pour ces auteurs application d'engrais (Howeler, 2001).

Le test de comparaison multiple de Dunnett qui consiste à faire la comparison entre le témoin $\left(\mathrm{T}_{0}\right)$ et les autres traitements a montré : i) des effets trés significatifs de l'apport de $\mathrm{N}$ issu de l'urée et de l'apport de potassium $(\mathrm{K})$ issu du sulfate de potassium $\left(\mathrm{K}_{2} \mathrm{SO}_{4}\right)$ sur le rendement en poids et en nombre de tubercules frais, de même que sur la croissance des plants du manioc; ii) un effet d'apports entre $\mathrm{N}$ et $\mathrm{K}\left(\mathrm{T}_{3}\right.$ et $\left.\mathrm{T}_{4}\right)$; de même entre $N$, P et $K\left(T_{8}, T_{9}\right.$ et $\left.T_{10}\right)$ sur le rendement au cours de cette étude. Par contre pas d'effet d'apports de $K$ et $P$ sur le rendement du manioc $\left(T_{5}, T_{6}, T_{7}\right.$, $T_{12}$ et $T_{13}$, sauf $T_{11}$ ). Ces résultats sont en accord avec les conclusions des travaux mené par Mitscherlich, agronome allemand (Mitscherlich, 1960).

Il est donc probable que l'augmentation de 21,50 t.ha ${ }^{-1}$ entre le témoin $\left(\mathrm{T}_{0}\right)$ et le meilleur traitement obtenu $\left(\mathrm{T}_{10}\right)$ soit due aux effets conjugués des trois éléments $N$, P et $K\left(T_{8}, T_{9}\right.$, et $\left.T_{10}\right)$. Des résultats 
similaires ont été obtenus en Côte d'Ivoire sur le manioc et dans d'autres pays, que les rendements les plus élevés correspondaient aux traitements ayant des apports simultanés de N, P et K (Bakayoko et al., 2009; Borah et al., 2010; Howeler, 1985; Noble, 2000; Pouzet, 1988; Pypers et al., 2011; Zoundi, 2012).

La dose d'application recommandée pour la culture de manioc est de : $67-\mathrm{N}+38-\mathrm{P}+122-\mathrm{K}$ (avec un ratio en $\mathrm{N}-\mathrm{P}-\mathrm{K}=1-2-3$ ) avec un rendement de tubercules frais de 37 t.ha $^{-1}$ (Howeler, 1985). Au vue de cette recommandation et selon nos résultats d'étude, on pourrait dire que la dose d’application qui réponde à la préférence des producteurs du manioc et d'avoir un bon rendement variant entre 30 et 40 t.ha ${ }^{-1}$ en tubercules frais du manioc, serrait la combinaison $80 \mathrm{~kg} / \mathrm{ha}$ d'azote $(\mathrm{N})+40 \mathrm{~kg} / \mathrm{ha}$ phosphore $(\mathrm{P})+120 \mathrm{~kg} / \mathrm{ha}$ de potassium $(\mathrm{K})$, soit un ratio en $\mathrm{N}-\mathrm{P}-\mathrm{K}=1-2-3$. Cette dose retenue permettra non seulement d'éviter la polution du sol par les excès d'engrais chimiques, mais aussi de limiter le pouvoir d'achat des paysans producteurs et de faire un peu d'économie. La méthode d'application retenue est celle de «split-application» et consiste à appliquer au premier de la plantation : $1 / 3$ de la quantité d'urée $(\mathrm{N})$ et de potassium $(\mathrm{K})$, ensuite à 3 mois $1 / 3$ de la quantité d'urée $(\mathrm{N})$ et de potassium $(\mathrm{K})$; et enfin le dernier tiers au $5^{\text {ième }}$ mois de la plantation. Quand au phosphore $(\mathrm{P})$, c'est sa quantité totale a appliquée dès le premier traitement (Kokram et al., 1996).

En comparant, les résultat obtenus pour les traitements $\mathrm{T}_{5}, \mathrm{~T}_{6}$ et $\mathrm{T}_{7}$ (avec apport de $\mathrm{K}_{2} \mathrm{SO}_{4}$ ) à ceux obtenus pour les traitements $\mathrm{T}_{11}, \mathrm{~T}_{12}$ et $\mathrm{T}_{13}$ (apport de $\mathrm{KCl}$ ), d'une part, on constate que les apports de potassium (K) sous forme de Chlorure de potassium $(\mathrm{KCl})$ ont tendance, au delà de 40 $\mathrm{kg} / \mathrm{ha}$, à réduire le rendement en poids et en nombre des tubercules frais du manioc par rapport aux apports de $\mathrm{K}$ sous forme de $\mathrm{K}_{2} \mathrm{SO}_{4}$. D'autre part , on observe que plus la dose de $\mathrm{KCl}$ augmente, plus le rendement diminue , alors que la tendance est inverse pour l'apport de $\mathrm{K}_{2} \mathrm{SO}_{4}$ (augmentation de la croissance des plants du manioc et du rendement en tubercules frais de manioc lorsqu'on augmente la dose d'application de $\mathrm{K}$ sous forme de sulfate de potassium $\left(\mathrm{K}_{2} \mathrm{SO}_{4}\right)$. Le sulfate de potassium $\left(\mathrm{K}_{2} \mathrm{SO}_{4}\right)$ serait donc mieux indiqué pour la fertilisation minérale du manioc au cours des études ultérieures.

Les résultats d'enquête réalisés auprès des paysans producteurs et des universitaires sur la l'appréciation de la qualité des tubercules crus et de la boule de manioc ont montré qu'ils n’y a pas d'effet des fumures minérales sur la qualité organoleptiques de manioc après la fertilisation. On a constaté que le pourcentage de participation de femme est plus élevé par rapport aux hommes. Cela peut s'expliquer par le faite que, le procédé de transformation de tubercule de manioc en cossette est souvent réservé aux femmes et se justifie aussi par le fait qu'elles sont très actives aux travaux de récole, de 
transformation, de la vente de manioc et ce qui fait qu'elles connaissent mieux la bonne qualité de manioc.

Selon les résultats d'enquête menés au près des femmes au cours de nos travaux, la couleur blanche et jaunâtre montre que le manioc est bien roui et bien séché ; le goût acidulé prouve la durée du pressage (48 heures) et la bonne qualité du manioc; ensuite une odeur agréable due à la bonne extraction du suc amer et au bon traitement durant le processus ; en plus la cossette agréable au toucher et facile à écraser et enfin la présence d'abeilles autour des cossettes de manioc.

\section{Conclusion}

En somme, les carences en certains minéraux de sol enrégistrées ont permis d'expliquer l'effet significatif des apports des engrais NPK sur le rendement moyen de $25 \mathrm{t}$. ha ${ }^{-1}$.

L’application des engrais minéraux a permis d’améliorer le rendment du manioc de 18,70 t.ha ${ }^{-1}$ pour le témoin non traité $\left(\mathrm{T}_{0}\right)$ à 40,20 t. ha ${ }^{-1}$ pour le meilleur traitement obtenu $\left(\mathrm{T}_{10}\right)$ au cours de cette étude. Elle a permis d'accroître la productivité du manioc avec une différence de 21,30 t.ha ${ }^{-1}$ entre le traitement témoin $\left(\mathrm{T}_{0}\right)$ et le meilleur traitement $\left(\mathrm{T}_{10}\right)$. Il y a une corrélation entre le rendement en tubercules frais et la croissance des plants du manioc. Cependant les formes de fumure testées ne sont pas équivalentes. Ainsi, si l'apport d'azote sous forme d'urée et de potassium sous forme de Sulfate de Potassium $\left(\mathrm{K}_{2} \mathrm{SO}_{4}\right)$ ont eu un effet positif sur le rendement du manioc, l'emploi de chlorure de potassium $(\mathrm{KCl})$, a eu un effet négatif à fortes doses.

Selon nos résultats, une fertilisation adéquate, qui réponde de mieux à la préférence des paysans producteurs et pour optimiser les rendements de la culture de manioc dans la zone de savane en Centrafrique, serait une combinaison NPK de $80 \mathrm{~kg} /$ ha d'urée $(\mathrm{N})$, de $40 \mathrm{~kg} / \mathrm{ha}$ de triple super phosphate TSP (P) et de $120 \mathrm{~kg} / \mathrm{ha}$ de $\mathrm{K}_{2} \mathrm{SO}_{4}$. Puisqu'elle permet non seulement d'éviter la polution du sol et de l'environement par les excès d'engrais chimiques, mais aussi de limiter le pouvoir d'achat des paysans producteurs.

Par ailleurs, selon l'appréciation des personnes ayant participé à l'enquête, l'application des engrais minéraux NPK n’a pas affecté la qualité organoleptique des tubercules cru et des boules de manioc.

Néanmoins, en perspective d'un développement plus durable en agriculture, d'autres pratiques restent à étudier pour éviter la dégradation des sols, notamment l'utilisation des terres de termitières comme engrais naturels et des légumineuses en association ou en rotation de culture. 


\section{Remerciements}

La présente étude a reçu l'appui financier de l'agence internationale de l'énergie atomique (AIEA-FAO) et du service de coopération et d'action culturelle français (SCAC). Les auteurs remercient le laboratoire LaSBAD de l’Université de Bangui-RCA, le laboratoire Eco\&Sols SupAgro-INRAIRD, Persyst US 49-CIRAD, Montpellier-France et le Laboratoire de Botanique Écologie Végétale (LBEV) de l’Université de Lomé-Togo pour leurs appuis techniques et scientifiques dans la réalisation de cette étude.

\section{References:}

Akanza, P., N'zue, B., and Anguete, K. (2002). Influence de la fumure minerale et de la litière de volaille sur la production du manioc (Manihot esculenta Crantz) en Cote d'Ivoire. Agronomie africaine 14, 79-89.

Backiny-Yetna, P., and Wodon, Q. (2010). "Profil et corrélats de la pauvreté en République Centrafricaine en 2008."

Baize, D. (2000). "Guide des analyses en pédologie: choix, expression, présentation, interprétation," Editions Quae.

Bakayoko, S., Tschannen, A., Nindjin, C., Dao, D., Girardin, O., and Assa, A. (2009). Impact of water stress on fresh tuber yield and dry matter content of cassava (Manihot esculenta Crantz) in Cte dIvoire. African Journal of Agricultural Research 4, 021-027.

Bois, C., and Baleux, F. (2003). Méthode de réalisation et intérêt d'un atlas numérique pour les acteurs du développement. In "Savanes africaines: des espaces en mutation, des acteurs face à de nouveaux défis. Actes du colloque, Garoua, Cameroun".

Borah, K. K., Bhuyan, B., and Sarma, H. (2010). Assessment of Soil Fertility Status in and Around the Tea Gardens of Undivided Darrang District, Assam. International Journal of Applied Environmental Sciences 5.

Buol, S. W., Southard, R. J., Graham, R. C., and McDaniel, P. A. (2011). "Soil genesis and classification," John Wiley \& Sons.

Doucet (2006). Guide pour l'interprétation d'une analyse de sol.

Giguère, R. (2002). "Botanique et horticulture dans les jardins du Québec: guide 2002," Éditions MultiMondes.

Giroux, M., and Audesse, P. (2004). Comparaison de deux méthodes de détermination des teneurs en carbone organique, en azote total et du rapport $\mathrm{C} / \mathrm{N}$ de divers amendements organiques et engrais de ferme. Agrosol 15, 107-110.

Howeler, R. (1985). Potassium nutrition of cassava. Potassium in agriculture, 819-841.

Howeler, R. (2011). Soil erosion control. 2011. The cassava handbook: A reference manual based on the Asian regional cassava training course, held 
in Thailand. International Center for Tropical Agriculture (CIAT), Cali, Colombia. p, 566-608.

Howeler, R. H. (1996). Diagnosis of nutritional disorders and soil fertility maintenance of cassava. Tropical Tuber Crops: Problems, Prospects and Future Strategies. Oxford and IBH Publishing Co., New Delhi, India, 181193.

Howeler, R. H. (2001). Nutrient Inputs and Losses in Cassava-based Cropping Systems-Examples from Vietnam and Thailand. Southeast Asia 20, 22.

Kafara, J.-M. (2003). Pratiques paysannes d'association de cultures dans les systèmes cotonniers des savanes centrafricaines. In "Savanes africaines: des espaces en mutation, des acteurs face à de nouveaux défis. Actes du colloque, Garoua, Cameroun".

Kaho, F., Yemefack, M., Feujio-Teguefouet, P., and Tchantchaouang, J. (2011). Effet combiné des feuilles de Tithonia diversifolia et des engrais inorganiques sur les rendements du maïs et les propriétés d'un sol ferralitique au Centre Cameroun. Tropicultura 29, 39-45.

Kokram, C., Donsay, M., Yampan, N., and Srijagcote, Y. (1996). "Study on sowing date of cowpea intercropped with cassava." Annual Research Report, Year 1996. Ubon Ratchathani Field Crops Research Center, Department of Agriculture, Ministry of Agriculture and Cooperatives.

La France, D., Leblanc, M., Gilbert, P.-A., Moreau, G., Lefebvre, M., Weill, A., Duval, J., Painchaud, J., and Houle, Y. (2012). "Mise au point et validation d'un système de travail minimum du sol avec planches permanentes en culture maraîchère biologique."

Milleville, P., and Serpantié, G. (1994). Dynamiques agraires et problématique de l'intensification de l'agriculture en Afrique soudanosahélienne $=$ Agrarian dynamics and the question of the intensification of farming in the Sahelian and savanna zones of Africa. Comptes Rendus de l'Académie d'Agriculture de France 80, 149-161.

Mitscherlich, A. (1960). "Medizin ohne Menschlichkeit: Dokumente des Nürnberger ärzteprozesses," Fischer-Taschenbuch-Verlag.

Moufti, A., and Mountadar, M. (2004). Lessivage des fluorures et des métaux à partir d'une cendre à charbon. Water quality research journal of Canada 39, 113-118.

Noble, B. F. (2000). Strategic environmental assessment: what is it? \& what makes it strategic? Journal of Environmental Assessment Policy and Management 2, 203-224.

Palm, C. A., Gachengo, C. N., Delve, R. J., Cadisch, G., and Giller, K. E. (2001). Organic inputs for soil fertility management in tropical agroecosystems: application of an organic resource database. Agriculture, ecosystems \& environment 83, 27-42. 
Pouzet, D. (1988). Improvements in the mechanized cultivation of cassava in the Ivory Coast. Amelioration de la culture mecanisee du manioc en Coted'Ivoire.

Pypers, P., Sanginga, J.-M., Kasereka, B., Walangululu, M., and Vanlauwe, B. (2011). Increased productivity through integrated soil fertility management in cassava-legume intercropping systems in the highlands of Sud-Kivu, DR Congo. Field crops research 120, 76-85.

Saragoni, H., Poss, R., Marquette, J., and Latrille, E. (1992). Fertilisation et succession des cultures vivrières au sud du Togo: synthèse d'une expérimentation de longue durée sur terres de barre. L'Agronomie tropicale 46, 107-120.

Staff, S. S. D. (1993). "Soil survey manual," United States Department of Agriculture.

Zinga, I., Chiroleu, F., Legg, J., Lefeuvre, P., Komba, E. K., Semballa, S., Yandia, S. P., Mandakombo, N. B., Reynaud, B., and Lett, J.-M. (2013). Epidemiological assessment of cassava mosaic disease in Central African Republic reveals the importance of mixed viral infection and poor health of plant cuttings. Crop Protection 44, 6-12.

Zinga, I., Harimalala, M., De Bruyn, A., Hoareau, M., Mandakombo, N., Reynaud, B., Lefeuvre, P., and Lett, J. (2012). East African cassava mosaic virus-Uganda (EACMV-UG) and African cassava mosaic virus (ACMV) reported for the first time in Central African Republic and Chad. New Dis. Rep., unpublished.

Zoundi, S. J. (2012). Agriculture vivrière: les Africains confrontés à des choix controversés de modèles agricoles. Cahiers Agricultures 21, 366-373. 